Research Article

\title{
Orthogonal Frequency Division Multiple Access Passive Optical Network for 5G Deployment
}

\author{
Pir Meher Ali Shah ${ }^{1 *}$, Shahryar Shafique Qureshi ${ }^{1}$, Rizwan Aslam Butt ${ }^{2}$, Haider Ali $^{3}$ and Shahid Khan ${ }^{4}$ \\ ${ }^{1}$ Department of Electrical Engineering, IQRA National University Peshawar, Khyber Pakbtunkbwa, Pakistan; ${ }^{2} N E D$ \\ University of Engineering and Technology, Karachi, Pakistan; ${ }^{3}$ Electrical and Electronics Engineering Technology Department, \\ University of Technology Nowshera, 24100, Pakistan; ${ }^{4}$ Institut Supérieur d'Électronique et d'Automatique, University De \\ Lorraine, Metz, Lorraine, 57070, France.
}

\begin{abstract}
Internet traffic and the number of devices is growing at an exponential rate. A robust communication network is required that provide high-capacity communication and integrate wired and wireless communication devices without creating bottlenecks. This work proposes a passive optical network (PON) architecture to ensure high-capacity paths between the densely deployed remote radio units (RRUs) with baseband units (BBUs) in $5 \mathrm{G}$ network architecture. Orthogonal frequency division multiple access (OFDMA) and wavelength division multiplexing (WDM) techniques are employed to ensure high capacity. Furthermore, free space optical (FSO) links are employed at the distribution level (DL) to facilitate dense deployment of RRUs near the subscriber's premises. The proposed model is analyzed in Optisystem, and it is observed to support 40 Gbps of data, over $4 \mathrm{~km}$ length of FSO.

Received: March 12, 2021; Accepted: June 24, 2021; Published: June 30, 2021

*Correspondence: Pir Meher Ali Shah, Department of Electrical Engineering, IQRA National University Peshawar, Khyber Pakhtunkhwa, Pakistan; Email: pirmeher@inu.edu.pk

Citation: Shah, P.M.A., S.S. Qureshi, R.A. Butt, H. Ali and S. Khan. 2021. Orthogonal frequency division multiple access passive optical network for $5 \mathrm{G}$ deployment. Journal of Engineering and Applied Sciences, 40(1): 37-43.

DOI: https://dx.doi.org/10.17582/journal.jeas/40.1.37.43

Keywords: Orthogonal frequency division multiple access (OFDMA), wavelength division multiplexing (WDM), passive optical network (PON), Free space optics (FSO), 5G
\end{abstract}

\section{Introduction}

$\mathrm{D}$ emand for high-capacity links have substantially grown over the past two decades owing to the development of low-cost communication devices that are connected to the Internet via numerous applications. Furthermore, adaptability of Internet of Things (IoTs) devices has opened a new pandora box of problems associated with the integration of many wired and wireless devices in the same network (Kulshreshtha and Garg, 2020).

The existing wireless communication technologies like $3 \mathrm{G}$ and $4 \mathrm{G}$ networks primarily utilize large cells to entertain the subscribers with acceptable data rates (Jaffer et al., 2020). However, such deployment of wireless networks might soon exhaust owing to the exponential growth of communication devices within a single cell (Esmail et al., 2019). Furthermore, wired technologies have the capacity to provide relatively high bandwidth, but they fail to facilitate wireless devices within the same network. Therefore, it is of primal importance to develop a network that can provide high capacity of wired networks and flexibility in connectivity of a range of devices like wireless networks, rely on base stations and large cells that cover a few kilometers of distance to provide connectivity. However, the current solution might soon exhaust owing to the exponential growth of devices and bandwidth requirement. In the said reference, the 
deployment of communication networks has shifted towards small cells that are deployed everywhere and connect devices to the core network (Zhang et al, 2017).

In a quest to find such a network, $5 \mathrm{G}$ communication systems are adapted that relies on small cell networks to facilitate large number of devices with high capacity in terms of data, reach and latency (Kulshreshtha and Garg, 2020). However, utilization of small cells requires the deployment of a dense communication network of base stations near the subscriber's premises. Consequently, $5 \mathrm{G}$ cell planning is expected to utilize and ultra-dense network of base stations with approximately 40-50 towers per Kilometer square (Esmail et al., 2019).

This cannot be achieved with effectively with the conventional architecture employed in the existing communication networks. $5 \mathrm{G}$ architecture employs the use of centralized Radio Access Networks (cRANs), which provides a cloud-based network architecture to facilitates the separation of base stations/ Remote Radio Units (RRU) from the Baseband units (BBU) and provides centralized BBUs. The connectivity between the BBU and the RRU is referred to as fronthaul networks as shown in Figure 1 (Jaffer et al., 2020).

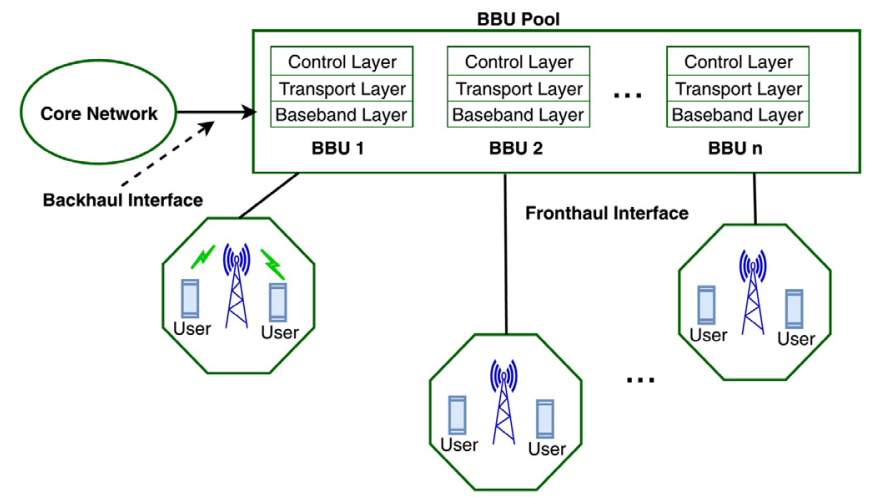

Figure 1: Connectivity options for $5 G$ networks [6].

Employment of cRAN and centralized BBUs simplifies the network architecture with the provision of low cost RRUs to be deployed at the subscriber's premises. However, to ensure high-capacity connectivity, a robust communication network is required that can provide the required bandwidth in between the centralized BBUs and corresponding RRUs (ITU-T 5G Standards, 2019).

In quest to solve the problem of a high-capacity net- work this work proposed an orthogonal frequency division multiple access (OFDMA) based passive optical network (PON). OFDMA is independently modulating subcarriers within frequencies (Bekkali et al., 2010). This method facilitates the use of simultaneous transmissions to and from multiple clients. Furthermore, wavelength division multiplexing (WDM) is utilized to facilitate the integration of transmission from multiple BBUs over the optical fiber and free space optical (FSO) channel (Li et al., 2021).

Performance of the proposed network architecture is evaluated in a highly recognized software called Optisystem. Analysis is made to determine the maximum transmission capacity by referring to the RF spectrum power at the receiving end and corresponding constellation points.

\section{Proposed architecture}

Existing solutions to the connectivity of centralized BBUs and RRUs essentially employs the utilization of dark fiber, passive WDM, and microwave links as show in in Figure 2. However, these solutions suffer from numerous drawbacks linked with high capital expenditure (CAPEX), complex network deployment, and utilization of low-capacity links that tend to create bottlenecks along the high-capacity links (Jaffer et al., 2020).

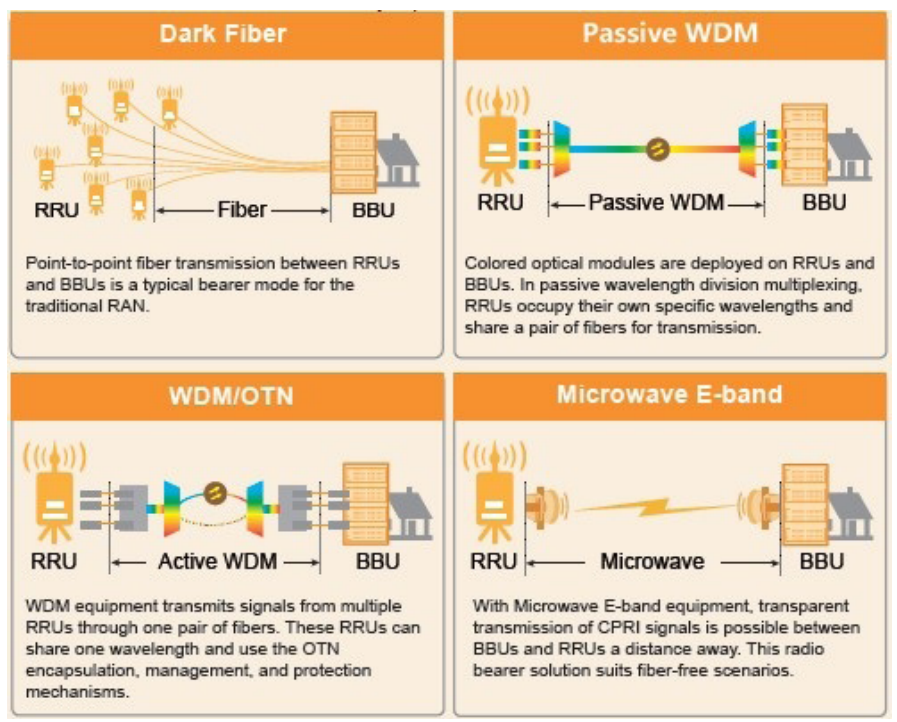

Figure 2: Connectivity solution between $B B U$ and $R R U$.

Consequent upon the above discussion, this work employs FSO based hybrid architecture for the deployment of ultra-dense $5 \mathrm{G}$ communication network. Optical fiber media is deployed at the feeder level that incorporates major span of the network. 
Whereas a combination of optical fiber media and FSO links is deployed at the distribution level as show in Figure 3 (ITU-T 5G Standards, 2019). Such an arrangement is employed to carry the traffic as close as possible to the subscriber's premises using a high-capacity optical fiber network. Whereas optical free space links are employed at the distribution level to facilitate dense deployment of RRUs at minimum complexity and CAPEX.
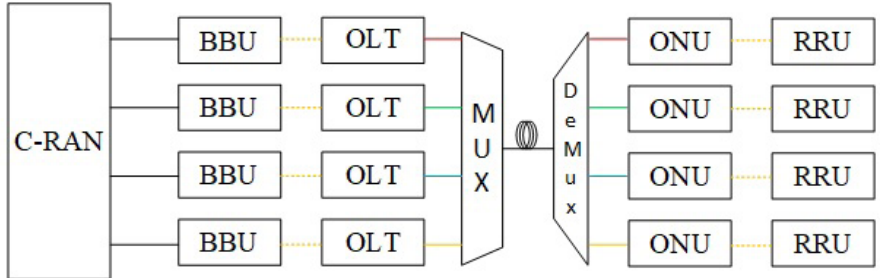

Figure 3: Lower layer split front haul based on dedicated $P O N$ architecture.

Figure 4 provides the PON architecture with OFDMA and WDM technologies for the deployment of $5 \mathrm{G}$ communication networks. The transmitter modules, representing BBUs, are formed by the combination of data source, quadrature phase shift keying (QPSK) sequence generator, OFDM modulation arrangement, RF-up-converter, and multiplexer (MUX) arrangement and laser diodes (LD).

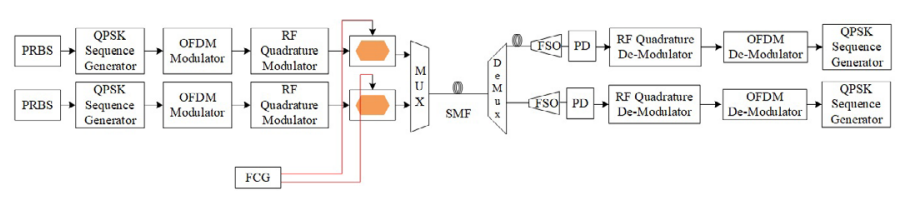

Figure 4: Proposed architecture for $B B U$ to $R R U$ connectivity.

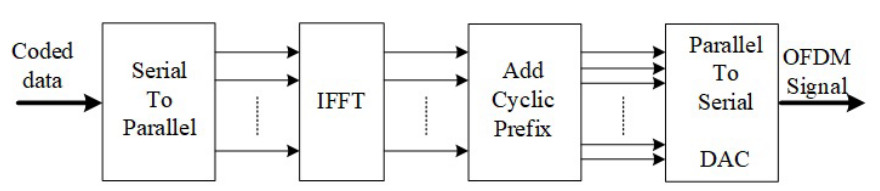

Figure 5: OFDM modulator operation blocks.

QPSK generator at the transmitter module is utilized to create 2 bits/ symbol to ensure spectral efficiency with and acceptable bit-error-rate (BER) of $2 \mathrm{e}^{-3}$. End-face of the QPSK signal generator is connected to an OFDM modulator as shown in Figure 5. The same is employed to convert the series stream of data into parallel for improve transmission capacity of the network. This step is followed by the utilization of an Inverse Fourier transform (IFFT) module, which converts the signal into time domain for the insertion of guard band along with the operations of modulation and multiplexing.

Output of the modulator is connected to RF-upconverter for the conversion of $10 \mathrm{Gbps}$ data signal from electrical to optical domain. The required optical carrier is provided with the help of LD as shown in Figure 4. The modulated optical signal is applied to WDM multiplexer (MUX) arrangement for transmission over the optical distribution network (ODN). Single mode fiber (SMF) is employed at the feeder level (FL) of PON to carry the multiplexed data from centralized BBU pool towards the respective RRU.

End-face of the SMF, deployed at the FL, terminated at the remote node (RN) to split the combined/ multiplexed signal from BBU pool. Corresponding data from each leg of the De-MUX arrangement is transmitted towards the RRU at the distribution level (DL). However, due to the congested deployment of RRUs, for high capacity and low latency, it is not possible to deploy an all fiber path towards each RRU. Consequently, FSO links are employed between the $\mathrm{RN}$ and corresponding RRU as shown in Figure 5.

\section{Analysis of the proposed architecture}

The proposed network architecture is analyzed for its performance in a highly recognized software application called Opti system. The simulation setup given in Figure 6, relies on the system model presented in Figure 4. Analysis is performed for four BBU and corresponding RRUs. It must be observed that each $\mathrm{BBU}$ and RRU is referenced as the transmitter and receiver modules of the proposed architecture.

\section{Simulation setup}

$10 \mathrm{Gbps}$ data is generated at each transmitter module through a 4-level ( 2 bits per symbol) QPSK sequence generator. OFDM modulator is employed after the QPSK generator to distribute the input bit sequence on to 512 subcarriers and 1024 FFT points. The real (I) and imaginary (Q) electrical constituents at output of each OFDM encoder are passed through a pulse shape low pass cosine roll-off filter with a rolloff factor of 0.2. The signals are then up converted to modulate at $10 \mathrm{GHz}$ with a CW laser diode (LD) in a window of 193.1-193.4 THz. Output of the WDM module is shown as Figure 7. Table 1 summarizes the parameters of system components used for the simulation analysis. 


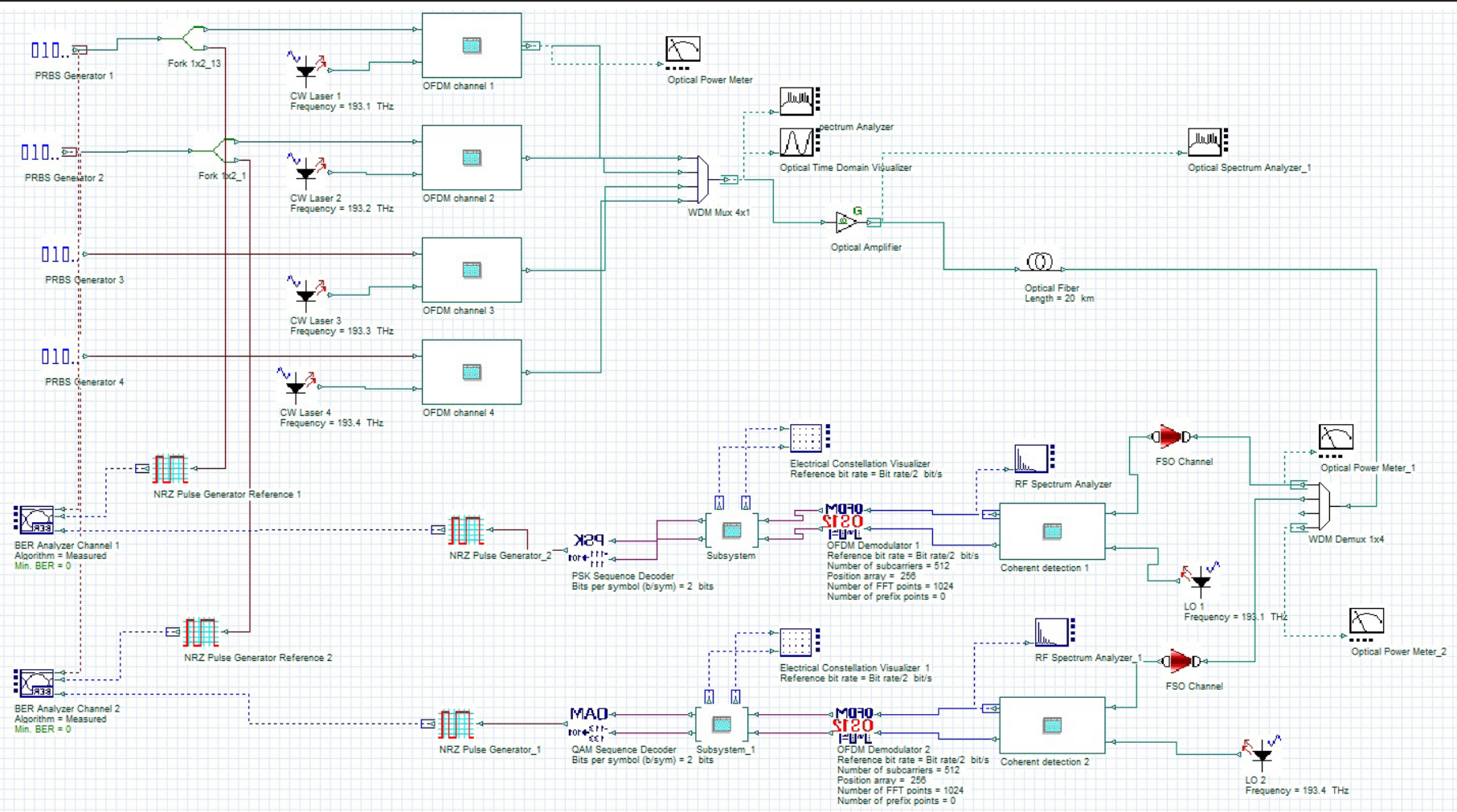

Figure 6: OFDMA-WDM PON implementation in Opti system.

目

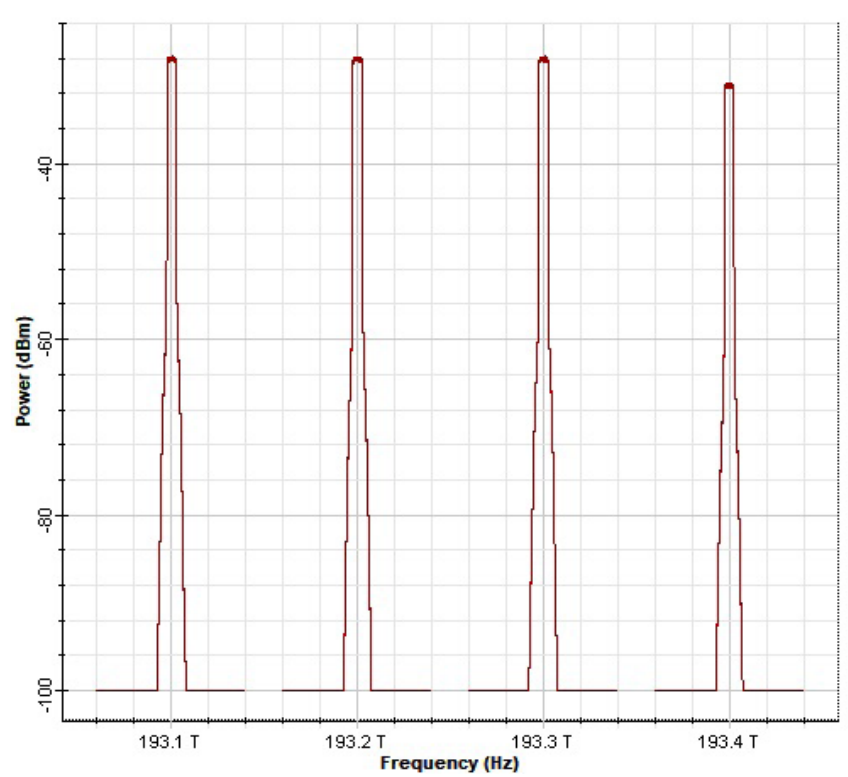

Figure 7: WDM multiplexed spectrum at the transmitter module.

Simulation result and analysis

Initial analysis is performed for the back-to-back (B2B) model in order to evaluate feasibility of the proposed architecture in terms of received RF spectrum and signal constellation. Figure 8 shows received RF spectrum and corresponding constellation points for a randomly selected node when four BBU modules are transmitting simultaneously at $10 \mathrm{Gbps}$ of data each.

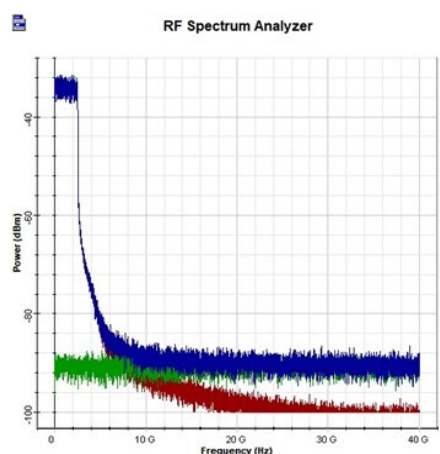

A

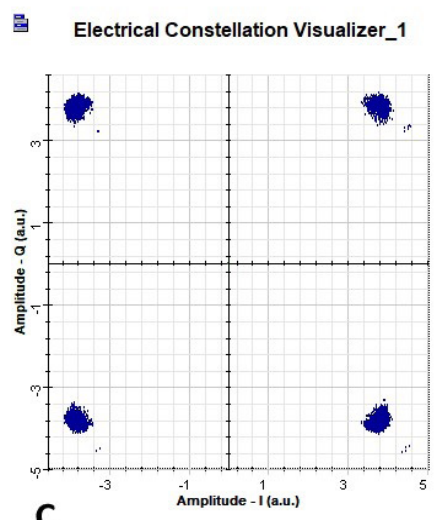

C

Figure 8: $R F$ spectrum and constellation points from B2B model at $-20 \mathrm{~dB}$ (a) (c) and $-25 \mathrm{dBm}$ (b) (d) received power respectively.

Figure $8 \mathrm{~A}$ and $8 \mathrm{~B}$ shows $\mathrm{RF}$ spectrum received at the end-face of the coherent detection scheme. It can be observed that the spectrum is effectively received with some bits noise (green wave). However, it is evident 
from the spectrum that power of the noise bits is significantly low in comparison with the intended spectrum. Figure $8 \mathrm{C}$ and $8 \mathrm{D}$ shows the received constellation points at $-20 \mathrm{dBm}$ and $-25 \mathrm{dBm}$ received power. The figure indicates good performance in terms of the constellation points where there are no points are received in error.

Table 1: System parameters and corresponding values.

\begin{tabular}{ll}
\hline Parameters & Values \\
\hline Modulation & OFDM QPSK \\
Subcarriers per band & 512 \\
Data rate per $\lambda$ & $10 \mathrm{Gbps}$ \\
Low-pass filter roll-off factor & 0.2 \\
MUX filter bandwidth & $10 \mathrm{GHz}$ \\
SMF length & $20 \mathrm{~km}$ \\
SMF attenuation & $0.25 \mathrm{~dB} / \mathrm{km}$ \\
SMF dispersion & $18 \mathrm{ps} / \mathrm{nm} / \mathrm{km}$ \\
SMF non-linear parameters & Active \\
FSO transmitter aperture & $5 \mathrm{~cm}$ \\
FSO receiver aperture & $20 \mathrm{~cm}$ \\
EDFA & $25 \mathrm{~dB} / \mathrm{km}$ \\
MZM extinction ratio & $60 \mathrm{~dB}$ \\
Cosine roll-off filters cutoff frequency & $0.62 *$ Bit rate \\
MUX/ De-MUX filter order & 2 \\
EDFA noise figure & $5 \mathrm{~dB}$ \\
Photodiode responsitivity & $0.75 \mathrm{~A} / \mathrm{W}$ \\
Photodiode noises & Dark, Thermal, Shot \\
\hline
\end{tabular}

For further analysis FSO link is introduced between the transmitter and receiver modules, and performance of the system is analyzed at $10 \mathrm{Gbps}$ of data for each BBU transmitter module. Validation results of the receiving module are demonstrated as $R F$ spectrum and corresponding constellation points as shown in Figure 9 at different lengths of the FSO media.

It can be observed from Figure 9 that performance of the system deteriorates as length of the FSO media increases. This is be evident from the fact that value of the signal power, in comparison with the noise generated at the receiving end, at the coherent detector deteriorates as length of the transmission media increases. This results in lower signal-to-noise ratio and elevates the value of bit error. The same can be observed from constellation points, where the noise of constellation is increasing with increase in length of the FSO media. Furthermore, Figure 10 also demonstrate the same behavior where strength of the signal deteriorates along with increase in length of the FSO channel. According to result of constellation visualizer, the proposed system is able to support upto $40 \mathrm{Gbps}$ of data, $10 \mathrm{Gbps}$ per BBU, with QPSK sequence bit is able to achieve $4 \mathrm{Km}$ length of FSO medium with maximum for 300 a.u and minimum for -300 a.u.
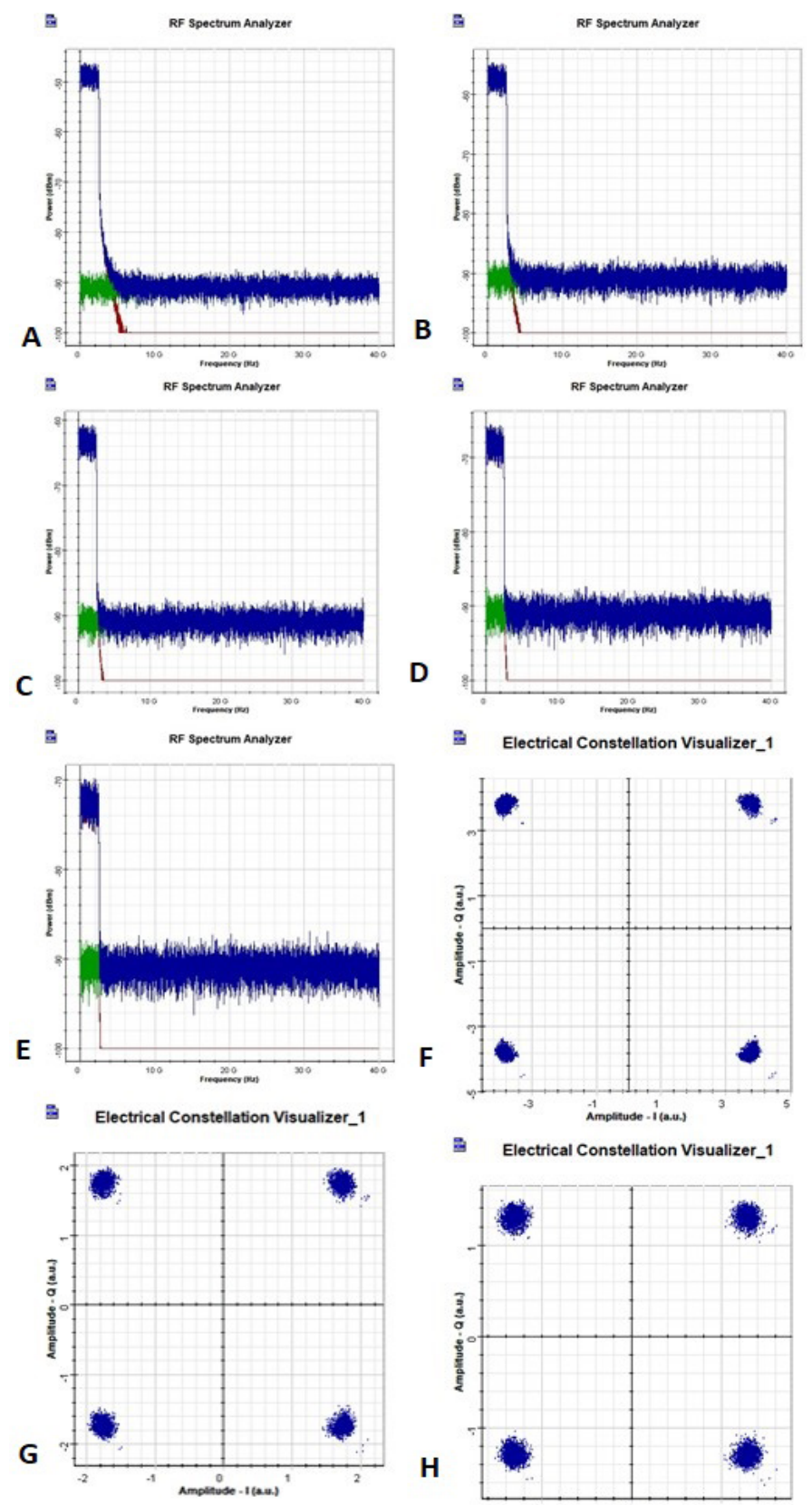

를 Electrical Constellation Visualizer_ 1
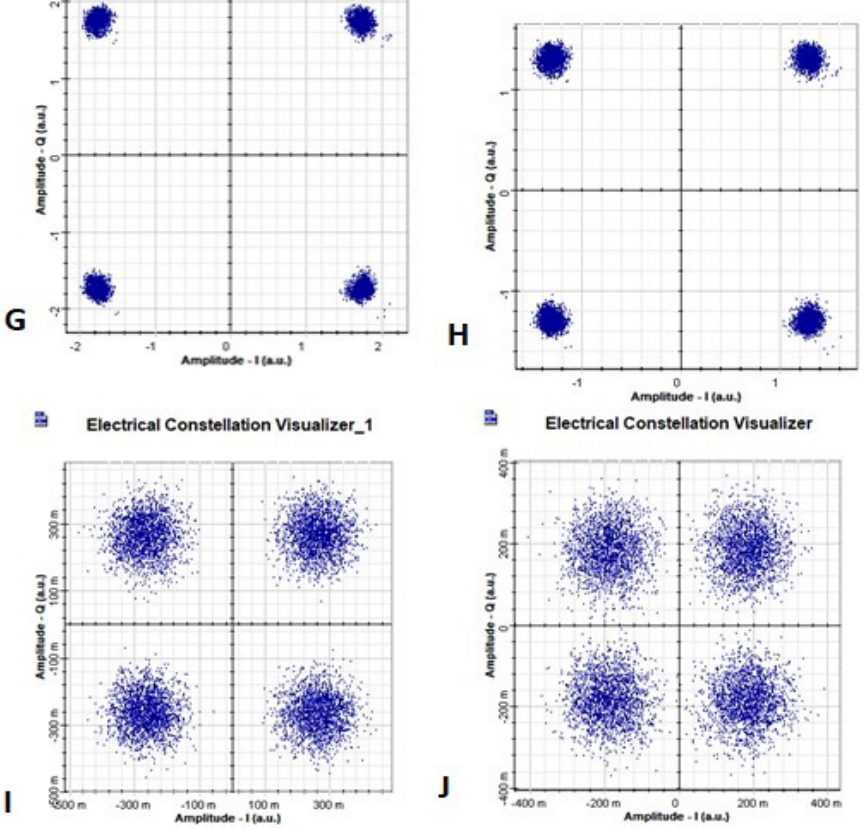

Figure 9: $R F$ spectrum and constellation points for FSO model at $2.5 \mathrm{~dB} / \mathrm{km}$ attenuation and $1 \mathrm{~km}(\mathrm{a})(f), 2 \mathrm{~km}(\mathrm{~b})$ (g), $3 \mathrm{~km}(\mathrm{c})(\mathrm{b}), 4 \mathrm{~km}(\mathrm{~d})(\mathrm{i})$, and $5 \mathrm{~km}$ (e) (j) FSO link. 


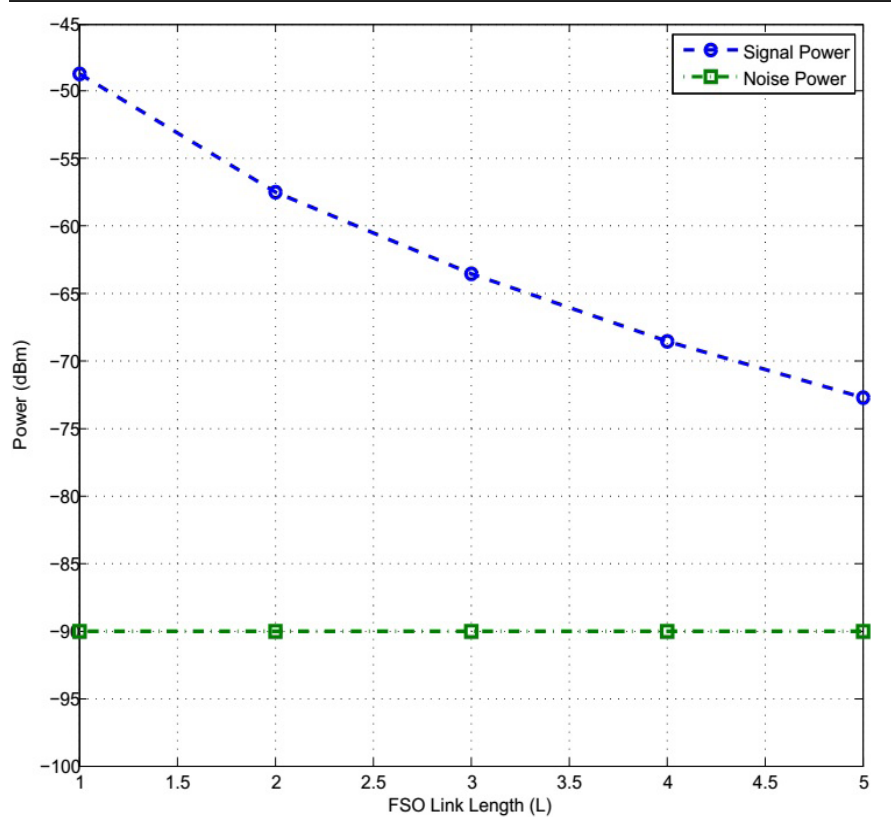

Figure 10: Signal power vs. noise power for different FSO link lengths.

\section{Conclusions and Recommendations}

This work proposed a network architecture for the deployment of $5 \mathrm{G}$ communication network with centralized BBU pool and dense RRUs. OFDMA is utilized to ensure high-capacity data transmission over PON architecture. Furthermore, FSO links are deployed at the distribution level to facilitate the necessary deployment at minimum complexity and cost of deployment. Simulation analysis via Opti system shows that the proposed architecture is well enough to support $10 \mathrm{Gbps}$ connectivity between $\mathrm{BBU}$ and corresponding RRU over the $4 \mathrm{Km}$ FSO link. Therefore, it can be concluded that the proposed FSO based PON architecture with OFDMA and WDM is able to support high-capacity connectivity in terms of data, reach, and the number of subscribers for the deployment of $5 \mathrm{G}$ communication network.

\section{Novelty Statement}

This paper proposes an orthogonal frequency division multiple access (OFDMA) based passive optical network (PON) architecture for the deployment of $5 \mathrm{G}$ network, which is optimized by the employment of frequency comb generator (FCG) at the BBU transmitter module and free space optical (FSO) link at the distribution level. Performance analysis shows support for $40 \mathrm{Gbps}$ of data over $20 \mathrm{~km} \mathrm{SMF}$ followed by $3 \mathrm{~km}$ FSO link at the distribution level.

\section{Author's Contribution}

Pir Meher Ali Shah: Conceptualization, methodology, system implemen-tation, analysis, manuscript drafting.

Shahryar Shafique: Results analysis, figures and proof reading.

Rizwan Aslam But: Simulation mode implementation and analysis.

Haider Ali: Facilitation with FSO channel model.

Shahid Khan: Interpretation of results and analysis.

\section{Conflict of interest}

The authors have declared no conflict of interest.

\section{References}

Bekkali, A., 2010. Transmission analysis of OFDM-based wireless services over turbulent radio-on-FSO links modeled by Gamma-Gamma distribution. IRRR Photonics J., 2(3): 510-520. https://doi.org/10.1109/ JPHOT.2010.2050306

Esmail, M.A., A.M. Ragheb, H.A. Fathallah, M. Altamimi and S.A. Alshebeili. 2019. 5G-28 $\mathrm{GHz}$ signal transmission over hybrid all-optical FSO/RF link in dusty weather conditions. IEEE Access, 7: 24404-24410. https://doi. org/10.1109/ACCESS.2019.2900000

Jaffer, S.S., A. Hussain, M.A. Qureshi and W.S. Khawaja. 2020. Towards the shifting of $5 \mathrm{G}$ front haul traffic on passive optical network. Wireless Personal Commun., 112(3): 1549-1568. https://doi.org/10.1007/s11277-020-07115-6

Kulshreshtha, P. and A.K. Garg. 2020. Managing $5 \mathrm{G}$ networks- $\mathrm{A}$ review of FSO challenges and solutions. In $202011^{\text {th }}$ IEEE International Conference on Computing, Communication and Networking Technologies (ICCCNT), 2020; 1-4. (1). https://doi.org/10.1109/ICCCNT49239.2020.9225591

Li, C.Y., H.H. Lu, C.R. Chou, H.M. Hsia, C.Y. Feng, Y.H. Chen and A. Nainggolan. 2021. A flexible bidirectional fiber-FSO-5G wireless convergent system. J. Lightwave Technol., 39(5): 1296-1305. https://doi.org/10.1109/ JLT.2020.3037943

Sup66, I.R.G., 2019. 5G wireless fronthaul requirements in a PON context. ITU-T Stan- 
open 2 access

dards, 2019.

Zhang, H., Y. Dong, J. Cheng, M.J. Hossain and

V.C. Leung. 2016. Fronthauling for 5G LTE-U

ultra-dense cloud small cell networks. IEEE

Wireless Commun., 23(6): 48-53. https://doi.

org/10.1109/MWC.2016.1600066WC 